\title{
CONTENÇÃO MECÂNICA: PERCEÇÃO DOS ENFERMEIROS
}

\author{
Mechanical restraint: Nurse`s perception \\ Contención mecánica: percepción de los Enfermeros
}

\author{
António Rodrigues ${ }^{*}$, Lúcia Gonzaléz ${ }^{* *}$, Pedro Castro ${ }^{* * *}$, Ricardo Silva ${ }^{* * * *}$, Ricardo Teixeira ${ }^{* * * *}$, Sérgio Martins ${ }^{* * * * * *}$, \\ Liliana Mota $^{* * * * * * *}$, Fernanda Príncipe ${ }^{* * * * * * * *}$
}

\section{RESUMO}

Enquadramento: a contenção mecânica é uma prática frequentemente utilizada pelos enfermeiros nos hospitais. O recurso à contenção está associado a problemas físicos, psicológicos, éticos e legais. Objetivos: identificar os conhecimentos e a prática dos enfermeiros sobre contenção mecânica; identificar a representação social da contenção mecânica para os enfermeiros. Metodologia: estudo qualitativo, do tipo exploratório, com uma amostra de 250 enfermeiros que responderam a um questionário elaborado no programa Google Forms. O tratamento e análise de dados foi efetuado no Software IRAMUTEQ. Resultados: mais de metade dos enfermeiros não conhece a orientação da Direção Geral de Saúde relativa à contenção de doentes e poucos têm formação específica, embora a pratiquem com frequência e sem intercorrências. Muitas vezes recorrem a técnicas que a previnem. A análise de similitudes destaca no corpus textual a prevenção, segurança e imobilização, como pensamentos dos enfermeiros. A segurança assume o núcleo central nos pensamentos relacionados com o doente e a tristeza nos pensamentos relativos à família. Conclusão: os enfermeiros praticam frequentemente a contenção mecânica por segurança, mas esforçam-se por evitá-la. Neste processo, valorizam mais os pensamentos dos familiares que os dos doentes.

Palavras-chave: imobilização; paciente; enfermeiro; prática

"Enfermeiro Especialista em Enfermagem MédicoCirúrgica, Centro Hospitalar Universitário do Porto. https://orcid.org/0000-0001-7051-6801

"*nfermeiro Especialista em Enfermagem MédicoCirúrgica, Centro Hospitalar Entre Douro e Vouga. https://orcid.org/0000-0003-4133-9310

***Enfermeiro Especialista em Enfermagem MédicoCirúrgica, Centro Hospitalar Entre Douro e Vouga. https://orcid.org/0000-0003-0658-3270

*... Enfermeiro Especialista em Enfermagem MédicoCirúrgica, Centro Hospitalar Universitário do Porto. https://orcid.org/0000-0001-9853-0277

".....Enfermeiro Especialista em Enfermagem Médico-cirúrgica, Centro Hospitalar Universitário do São João. https://orcid.org/0000-0002-9590-6352

*......Enfermeiro Especialista em Enfermagem Médico-Cirúrgica, Centro Hospitalar Universitário do São João. https://orcid.org/0000-0002-5485-8200

.......* PhD, Professora Adjunta na Escola Superior de Cruz Vermelha Portuguesa/CINTESIS. https://orcid.org/0000-00033357-7984

******* PhD, Professora Coordenadora na Escola Superior de Saúde Norte da Cruz Vermelha Portuguesa/CINTESIS. https://orcid.org/0000-00021142-3258

Como Referenciar:

Rodrigues, A. et al. (2020). Contenção mecânica: perceção dos enfermeiros. Revista de Investigação \& Inovação em Saúde, 3(1), 31-41

\section{ABSTRACT}

Background: mechanical restraint is a practice widely used by nurses in hospitals. The use of mechanical restraint is associated with physical, psycological, legal and ethical problems. Objectives: to identify nurses knowledge and practice on mechanical restraint; Identify the social representation of mechanical restraint for nurses. Methodology: qualitative, exploratory study with a sample of 250 nurses who answered a questionnaire prepared in the Google Forms program. Data treatment and analysis was performed using the IRAMUTEQ Software. Results: more than half of the nurses do not know the General Direction of Health guidance on patient containment and few have specific training, although they often practice uneventfully. They often resort to techniques that prevent it. The analysis of similarities highlights in the textual corpus prevention, safety and immobilization, as thoughts of nurses. Safety takes center stage in thoughts related to the patient and sadness in feelings concerning the family. Conclusion: nurses often practice mechanical restraint for safety, but strive to avoid it. In this process, they value the feelings of family members more than those of patients.

Keywords: restrains; patient; nurse; practice

\section{RESUMEN}

Marco contextual: la contención mecánica es una práctica frecuentemente utilizada por los enfermeros en los hospitales. El uso de restricciones mecânicas está associado com problemas físicos, psicológicos, éticos y legales. Objetivos: identificar los conocimientos y la práctica de los enfermeros sobre contención mecánica; identificar la representación social de la contención mecánica para los enfermeros. Metodología: estudio cualitativo, del tipo exploratorio, con una muestra de 250 enfermeros que respondieron a un cuestionario elaborado en el programa Google Forms. El tratamiento y análisis de los datos ha sido efectuado en el Software IRAMUTEQ. Resultados: más de la mitad de los enfermeros no conoce la orientación de la Dirección General de Salud relativa a la contención de pacientes y pocos tienen formación específica, aunque la practiquen con frecuencia sin complicaciones. Muchas veces recurren a técnicas que lo impiden. El análisis de similitudes destaca en el cuerpo textual la prevención, seguridad e inmovilización, como pensamientos de los enfermeros. La seguridad asume el núcleo central en los pensamientos relacionados com el paciente y la tristeza en los sentimientos relativos a la familia. Conclusión: los enfermeros practican frecuentemente la contención mecánica por seguridad, pero se esfuerzan por evitarla. En este proceso, valorizan mas los sentimientos de los familiares que los de los pacientes.

Palabras clave: inmovilización; paciente; enfermeiro; practica 


\section{INTRODUÇÃO}

Durante a prestação de cuidados que objetivam o tratamento e a melhoria da saúde do doente, existe por vezes a necessidade de recurso a técnicas de contenção mecânica para garantia da segurança. A contenção mecânica é uma intervenção frequente dos enfermeiros, apresentando uma taxa de prevalência entre $33 \%$ e $68 \%$ nos hospitais (Hamers \& Huizing, 2005). Sendo estes profissionais os que estão mais próximos dos doentes caberá aos mesmos a função de proceder à contenção mecânica. A nível legal serão elegíveis os doentes que manifestem comportamentos que coloquem a si ou a sua envolvência em risco ou que recusem tratamento compulsivo, vital e urgente (DGS, 2011).

Foram desenvolvidos estudos (Paes, Borba, Maftum, 2011; Kontio, Valimaki, Putkonen, Kuosmanen, Scott, \& Joffe, 2010; Martin, \& Mathisen, 2005) em diversos países que procuraram retratar a prática e o desenvolvimento de métodos para uma redução da utilização de medidas de contenção de doentes. Em Portugal, apenas identificamos um estudo nesta área, desenvolvido por Faria, Paiva \& Marques (2012).

O pouco conhecimento da realidade portuguesa no que se refere à contenção mecânica é a razão da investigação que nos propusemos efetuar, com a qual pretendemos dar resposta à questão "Quais os conhecimentos sobre a contenção mecânica e sua representação social para os enfermeiros?"

Dirigimos o nosso estudo tendo como foco a perspetiva dos enfermeiros de diferentes serviços sobre a prática da contenção mecânica, com o objetivo de identificar os conhecimentos e a prática dos enfermeiros sobre contenção mecânica e identificar a representação social da contenção mecânica para os enfermeiros.

\section{ENQUADRAMENTO/ FUNDAMENTAÇÃO TEÓRICA}

Em 2011, a Direção Geral de Saúde (DGS) define contenção mecânica como a "utilização de instrumentos ou equipamentos que restringem os movimentos do doente" (p. 3).

Estudos realizados demonstram que as principais razões para o recurso a medidas de contenção mecânica são a segurança da pessoa e do ambiente envolvente, prevenção de quedas, prevenção da remoção de dispositivos médicos e para permitir um tratamento médico emergente (Fletcher \& Hirdes, 2010; Hamers \& Huizing, 2005; Choi, \& Song, 2003; Segatore \& Adams, 2001;)

Atualmente, existe um enfoque no recurso a medidas alternativas e de prevenção tais como a presença e acompanhamento individual por profissionais de saúde que proporcionem ao doente a libertação de tensões, comunicação sincera e firme, ambiente calmo e seguro, inclusão ou exclusão da pessoa significativa, organização de atividades compatíveis com a condição do doente e tratamento farmacológico (DGS, 2011). Vivenciamos o desenvolvimento das ciências sociais e o reconhecimento do código deontológico, que induzem nos profissionais de saúde a consideração de aspetos éticos que no passado eram descurados. A perspetiva paternalista da medicina, onde os profissionais de saúde decidiam o que é melhor para o doente, translada para uma cooperação onde os procedimentos e tratamentos médicos são negociados em colaboração com ele. 
Quando a segurança do tratamento e dos respetivos intervenientes fica comprometida por comportamentos manifestados pelo doente, a decisão de proceder a uma contenção mecânica é quase sempre do enfermeiro, por ser o profissional de saúde mais próximo do doente. A formação e experiência destes profissionais dotam-nos de competências técnicas e científicas que Ihes permitem intervir adequadamente.

A própria DGS (2011) reconhece a relevância do poder de decisão do enfermeiro no momento de proceder à contenção mecânica, revogando a circular normativa no 08/DSPSM/DSPCS (DGS, 2007) de 25/05/07 que referia o dever de "ser realizada sob prescrição médica" quando emite a orientação no 021/2011 de 06/06/2011 que não faz qualquer referência a este dever. Recomenda que a decisão seja discutida em grupo, mas de acordo com Kontio et al. (2010) a decisão de proceder a uma contenção tem frequentemente de ser tomada rapidamente e os enfermeiros não têm tempo suficiente para discutir com outros membros da equipa, especialmente com os médicos. Consequentemente, ficam com dúvidas acerca do procedimento que decidiram e se fizeram o suficiente para o prevenir, considerando as alternativas existentes.

É sobre os pensamentos que ocorrem aos enfermeiros no processo de instituir uma contenção mecânica que esta investigação incide. A ambivalência de pensamentos entre o direito à autodeterminação e a necessidade de decidirem o que é melhor para o doente, instituindo planos terapêuticos que visem o tratamento da doença e salvaguarda da saúde, geram inquietações que pretendemos aprofundar.
Em Portugal, a temática da contenção mecânica está pouco estudada. De acordo com o estudo de Faria, Paiva \& Marques (2012) a contenção mecânica "temse manifestado como uma intervenção do fenómeno confusão, que condiciona o processo do doente" (p. 8). A população deste estudo foi constituída por 552 doentes internados em vários serviços, e a amostra selecionada foi constituída por 110 doentes sujeitos à contenção (19,9\%). Das várias razões apontadas pelos enfermeiros para a aplicação desta medida, destacamse o risco de queda $(36,5 \%)$ e a prevenção da exteriorização de dispositivos médicos (20,2\%). Em $80 \%$ destes doentes não foi tentada qualquer alternativa prévia à contenção. Este estudo revela a existência de uma associação significativa entre contenção mecânica e doentes idosos, confusos, com alterações do equilíbrio, com patologia das vias respiratória e circulatória e totalmente dependentes no autocuidado.

Embora frequentemente considerado como procedimento aceitável da prática, o uso de contenção mecânica está associado a problemas físicos, psicológicos, éticos e legais (Martin \& Mathisen, 2005). Em Portugal, os enfermeiros exercem de acordo com os deveres do Código Deontológico. Dita o primeiro dos princípios gerais que "as intervenções de enfermagem são realizadas com a preocupação da defesa da liberdade e da dignidade da pessoa humana e do enfermeiro" (Código Deontológico dos Enfermeiros, 2015). Este código consagra também direitos aos membros da Ordem dos Enfermeiros, sendo um deles "usufruir de condições de trabalho que garantam o respeito pela deontologia da profissão e pelo direito dos cidadãos a cuidados de enfermagem 
de qualidade" (Código Deontológico dos Enfermeiros, 2015).

Pretendemos esclarecer como a contenção mecânica é praticada e compreendida pelos enfermeiros, assim como analisar a expressão dos seus pensamentos quando recorrem a este procedimento. Deste modo, suportamo-nos na Teoria das Representações Sociais para dar forma ao nosso estudo. Segundo Silva, Camargo \& Padilha (2011) a Teoria das Representações Sociais, desenvolvida por Moscovici na década de 50, é muito utilizada na área da enfermagem, "devido à possibilidade do investigador captar a interpretação dos próprios participantes da realidade que se almeja pesquisar, possibilitando a compreensão das atitudes e comportamentos que um determinado grupo social frente a um objeto psicossocial" (p. 948).

\section{METODOLOGIA}

Este estudo é qualitativo do tipo exploratório, uma vez que pretendemos estudar experiências humanas, no sentido de identificar os pensamentos dos enfermeiros associados à contenção mecânica dos doentes.

A população em estudo são os enfermeiros portugueses a exercer em instituições hospitalares, por ser a realidade dos investigadores e da maioria dos enfermeiros. O método de amostragem foi não probabilístico por conveniência, sendo que o limite temporal foi o elemento que condicionou o número de participantes. Como critério de exclusão definiu-se que não participariam no estudo os enfermeiros que desempenhassem funções em serviços não clínicos e consulta externa, por ausência de contacto direto com doentes ou contacto por tempo limitado. A amostra foi constituída por 250 enfermeiros que responderam ao questionário.

A colheita de dados decorreu entre março e maio de 2019, por meio de um questionário elaborado no Google Forms para ser facilmente difundido via e-mail e partilhado nas redes sociais. 0 referido questionário é constituído por duas partes. A primeira parte tem questões fechadas referentes às caraterísticas sociodemográficas, conhecimento da norma da DGS (2011), formação em serviço e prática sobre contenção mecânica. A segunda parte é constituída por questões abertas de resposta curta sobre os pensamentos dos enfermeiros em relação à prática da contenção mecânica. A construção da segunda parte do instrumento baseou-se na técnica da múltipla associação livre de palavras (TALP), com a elaboração de três perguntas indutoras de estímulo, pedindo ao participante que respondesse com quatro palavras ou expressões sem restrição de resposta. Os três estímulos indutores utilizados no TALP foram: contenção mecânica, doente e pensamento; através da apresentação de variações da expressão: "quando penso em...", "lembro de...". Este método carateriza-se por ser um teste projetivo, possibilitando a apreensão das representações cognitivas de um grupo social de forma espontânea, revelando também conteúdos implícitos ou latentes que podem ser dissimulados nas práticas discursivas. Estrutura-se pela evocação de respostas com base num estímulo indutor, permitindo evidenciar universos semânticos relacionados com um determinado objeto.

Para tratamento e análise dos dados foi utilizado o Software IRAMUTEQ - Interface de R pour les Analyses Multidimensionnelles de Textes et de Questionnaires, 
(versão 0,7 alpha 2). O IRAMUTEQ é um software gratuito e com fonte aberta, desenvolvido por Pierre Ratinaud, que permite fazer análises estatísticas sobre textos e sobre palavras.

Este estudo de investigação foi submetido à Unidade de Investigação e Desenvolvimento e Comissão de Ética da Escola Superior de Saúde Norte da Cruz Vermelha Portuguesa tendo o obtido parecer favorável (Parecer no 13/2019). A participação no estudo foi voluntária. A garantia da confidencialidade das informações e o anonimato dos participantes foi uma preocupação neste estudo, pelo que, no processo de recolha e análise dos dados, não constou qualquer elemento que permitisse identifica-los.

\section{RESULTADOS}

A maioria dos enfermeiros que participaram no estudo é do sexo feminino (72\%; $n=180$ ) e mais de metade são casados (56,8\%; $n=142)$. A idade média dos participantes é de $36,6 \pm 6,1$ anos, tendo o mais novo 24 e o mais velho 57 anos.

Relativamente às habilitações académicas $84 \%(n=210)$ são licenciados e $16 \%(n=40)$ têm o grau de mestre. Quanto ao local onde desempenham funções, destacam-se a urgência $(31,6 \% ; n=79)$, internamento $(25,2 \% ; n=63)$ e medicina intensiva $(16,4 \% ; n=41)$.

No que diz respeito ao tempo de exercício profissional, o tempo médio de experiência é de $14 \pm 6$ anos, variando entre 1 e 39 anos.

Mais de metade dos participantes 54\% ( $n=135)$ não conhece a orientação da DGS (2011) relativa à contenção de doentes. Apenas 9,6\% (n=24) dos enfermeiros referiu ter formação em serviço sobre contenção mecânica. E na globalidade da amostra
(70,4\%; n=176) considerou não ser adequada a sua formação sobre esta temática.

Quando necessitam de utilizar uma técnica de contenção, o método de eleição é o químico (47,2\%; $n=118)$, seguido da contenção mecânica (20\%; $n=50)$, ambiental $(16,8 \% ; n=42)$ e física $(16 \% ; n=40)$.

Quando questionados acerca da frequência com que necessitam de aplicar medidas de contenção mecânica $38,8 \%$ ( $n=97)$ dos enfermeiros refere ser uma intervenção frequente, $16,4 \%(n=41)$ muito frequente e $36,4 \% \quad(n=91)$ enfermeiros admitem fazê-lo raramente. A maioria dos enfermeiros, $82 \%(n=205)$, refere que nunca teve intercorrências durante a aplicação de contenção mecânica. Os restantes 18\% $(n=45)$ admitem intercorrências resultantes do procedimento, sendo as mais frequentes a solução de continuidade cutânea (31,1\%; n=14), a incompreensão dos familiares (20\%; $n=9)$, equimose $(20 \% ; n=9)$ e edema $(17,8 \% ; n=8)$.

Quanto à frequência da reavaliação da necessidade de contenção mecânica, 44\% $(n=110)$ dos enfermeiros realiza-a na primeira hora, $24,4 \%(n=61)$ na segunda hora e $10,4 \%(n=26)$ na terceira hora. 0 tempo médio para reavaliação é de 3,4 horas, com oscilação entre o intervalo de tempo 0,2 horas e 48 horas.

A maioria dos enfermeiros $(66,8 \% ; n=167)$ refere utilizar técnicas preventivas da contenção mecânica. As mais utilizadas são o diálogo com o doente $(37,7 \%$; $\mathrm{n}=57)$, a contenção química $(17,2 \% ; n=26)$, a contenção ambiental (14,6\%; $n=22)$, a orientação espacial e temporal (8,6\%; $n=13)$ e o envolvimento da família $(7,3 \% ; n=11)$.

Na análise da representação social, quando solicitados a expressão de 4 pensamentos relativos à contenção mecânica, os enfermeiros recorreram a 193 palavras 
diferentes. Destas, 23 foram referidas mais de 10 vezes

e 125 palavras foram referidas apenas uma ou duas vezes.

O programa IRAMUTEQ posiciona aleatoriamente as palavras para que as mais frequentes aparecem maiores que as outras, demonstrando assim o seu destaque no corpus da análise da pesquisa (Marchand \& Ratinaud, 2011).

Segurança foi o pensamento mais referido (32\%; $n=80)$, seguido de imobilização $(24,4 \% ; n=61)$, agitação (22,8\%; n=57), prevenção $(18,4 \% ; n=46)$ e amarrar
(18\%; $n=45)$. Estes cinco pensamentos mais referidos reúnem $28,9 \%$ do total de mil pensamentos. Os dez pensamentos mais descritos reúnem $42,9 \%$.

$\mathrm{Na}$ árvore de similitudes (figura 1), extraída do mesmo programa, visualiza-se a ligação entre as palavras do corpus textual. Os elementos com maior centralidade são a prevenção, a segurança e a imobilização. O elemento segurança ramifica-se em necessidade, agitação, proteção e risco. O elemento imobilização ramifica-se em amarrar, pulseira e contenção.

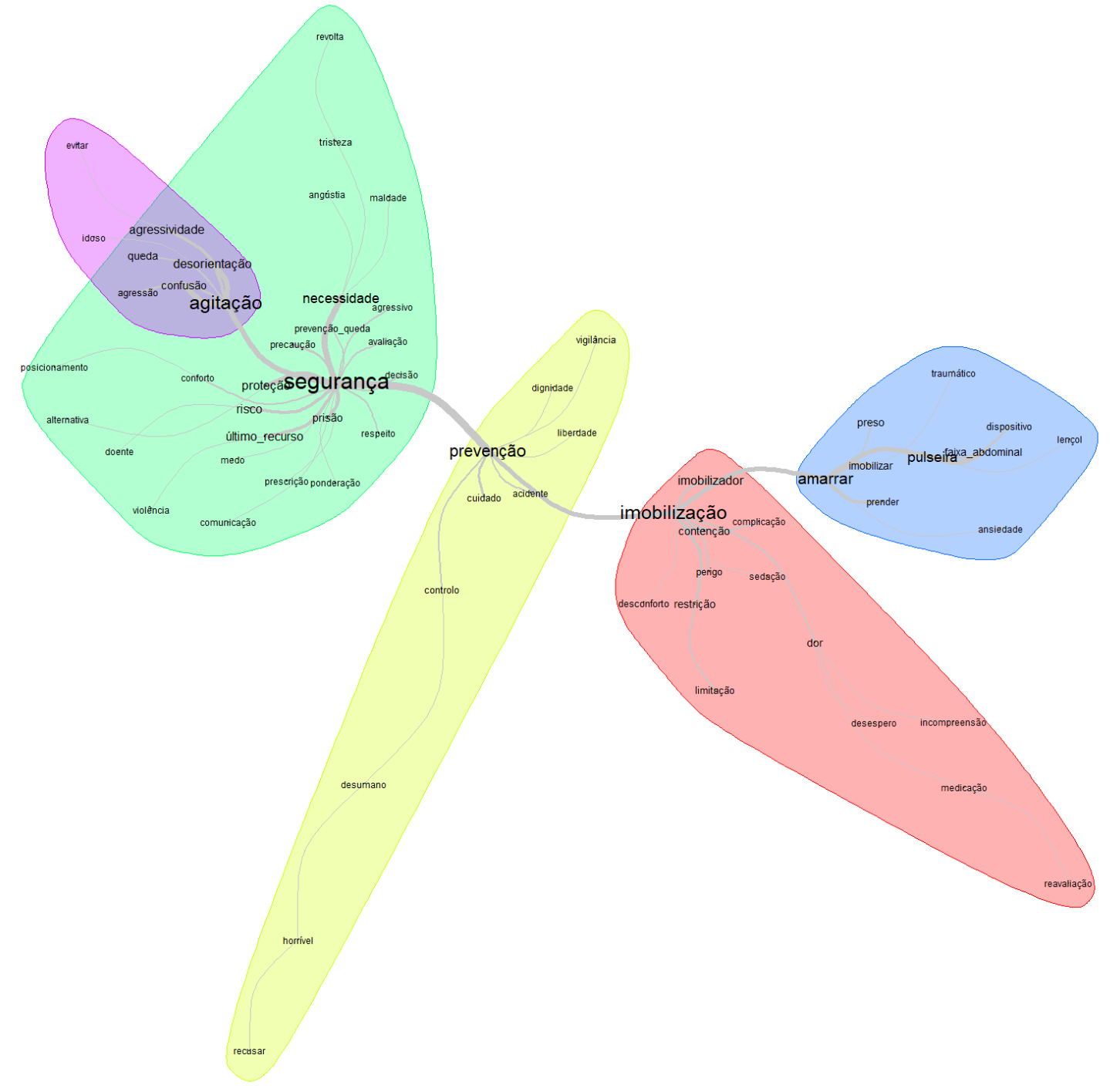

Figura 1

Árvore de similitudes: procedimento de contenção mecânica. 
Relativamente à perceção que os enfermeiros têm ( $n=67)$ prevenção, 18,4\% ( $n=46)$ último recurso e sobre os pensamentos dos doentes sujeitos à $13,6 \%(n=34)$ proteção. Estes cinco pensamentos mais contenção mecânica, foram descritas 242 palavras. referidos reunem 32,6\% do total de mil pensamentos. Destas 16 foram referidas mais de 10 vezes e 166 Os dez pensamentos mais descritos reúnem 40,6\%. Na palavras foram referidas apenas uma ou duas vezes. árvore de similitudes visualiza-se como elemento Quase metade dos enfermeiros $(44,4 \% ; n=111)$ central a segurança (figura 2). Desta ramificam a referiram segurança, 27,2\% ( $n=68$ ) necessidade, 26,8\% prevenção, proteção, último recurso e necessidade.

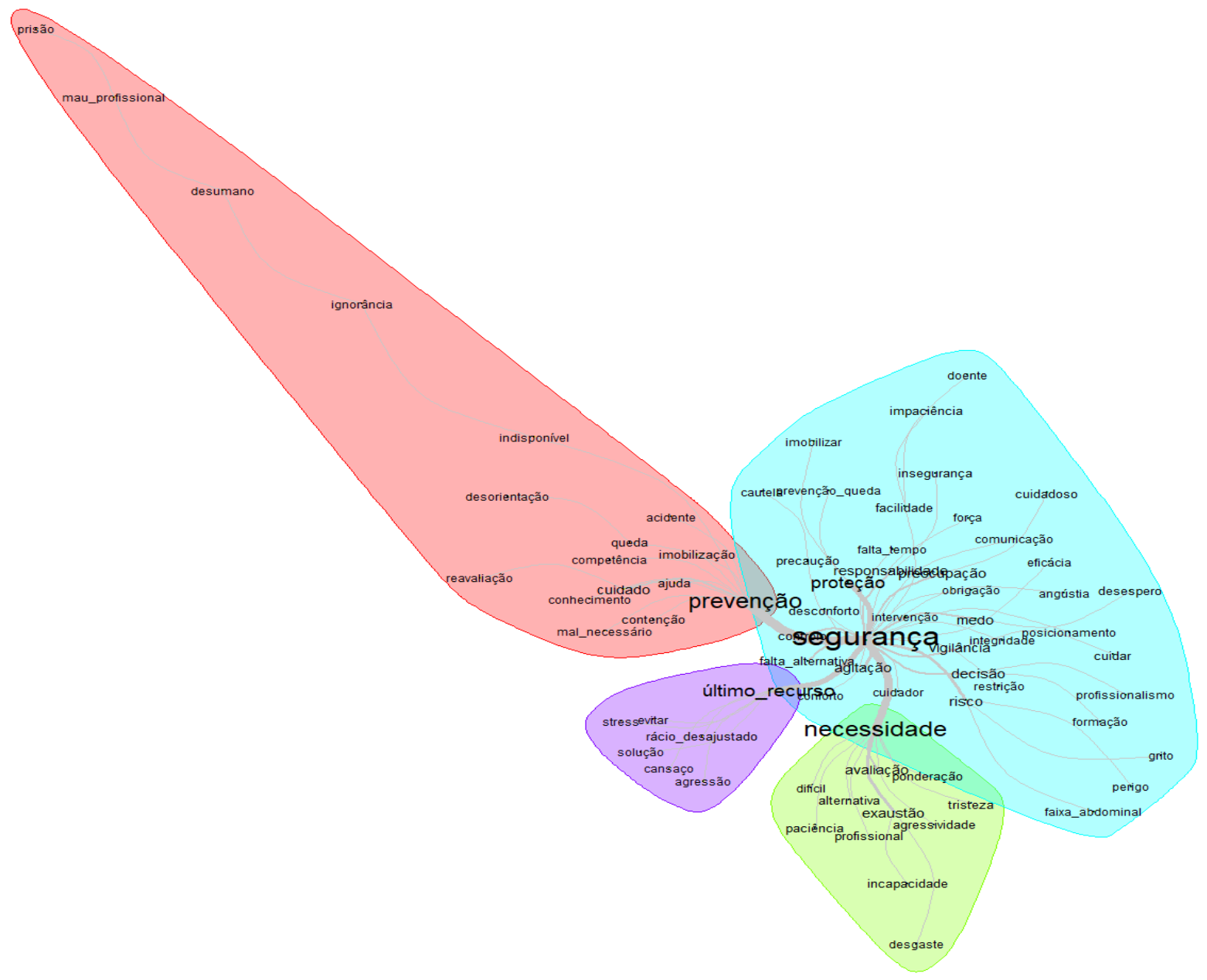

Figura 2

Árvore de similitudes: enfermeiros que utilizam a contenção mecânica

No que diz respeito à perceção que os enfermeiros têm sobre os pensamentos dos familiares dos doentes sujeitos à contenção mecânica, foram referidas 224 palavras. Destas, 17 foram referidas mais de 10 vezes e 150 palavras foram referidas apenas uma ou duas vezes.

Tristeza foi a palavra mais referida, escolhida por $37,2 \% \quad(n=93), \quad 26 \% \quad(n=65) \quad$ responderam 
incompreensão, 22,4\% ( $n=56)$ revolta, 17,2\% ( $n=43)$ último recurso e $14 \%(n=35)$ necessidade. Estes cinco pensamentos mais descritos representam $29,2 \%$ do total de mil pensamentos.
Os dez pensamentos mais referidos representam $42,6 \%$. Na árvore de similitudes visualizam-se como elementos centrais a tristeza, a incompreensão e a revolta (figura 3).

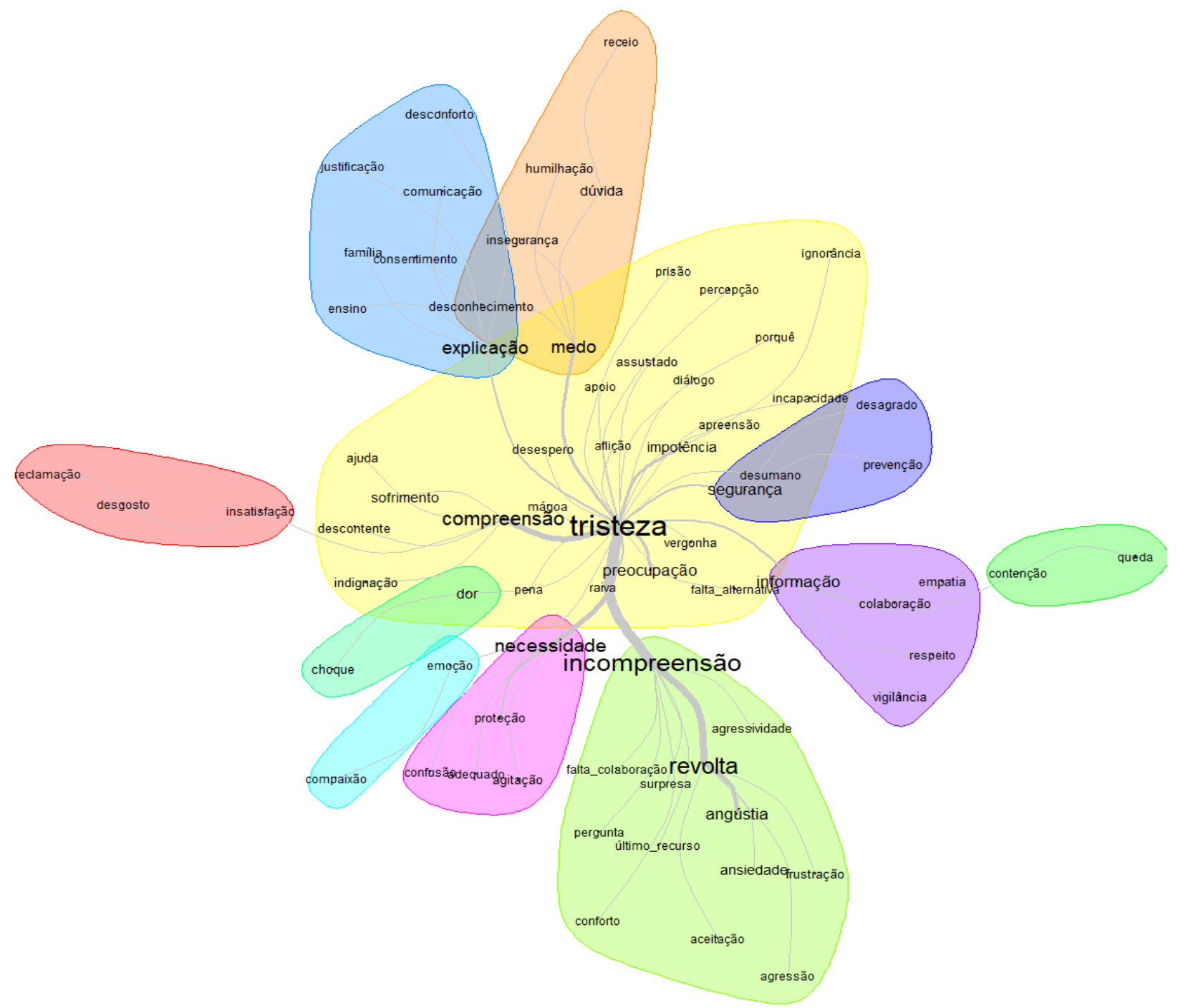

Figura 3

Árvore de similitudes: familiares de doentes em contenção mecânica 
A análise de similitude permitiu visualizar a relação entre as palavras e a sua conectividade dentro de cada classe e por outro lado a ligação entre as várias classes (Marchand \& Ratinaud, 2011).

\section{DISCUSSÃO}

$\mathrm{Na}$ interpretação dos resultados sobre o conhecimento que os enfermeiros têm sobre a norma da DGS (2011) relativa à contenção de doentes, verificamos que sensivelmente metade dos enfermeiros não a conhece. Apuramos também que o número de enfermeiros que teve formação em serviço sobre contenção mecânica não chega aos 10\%, embora um terço considere possuir formação adequada sobre a intervenção.

Das quatro formas de contenção, quase metade dos enfermeiros privilegiam a química e apenas um quinto privilegia a mecânica. No entanto, a maioria (55,6\%; $\mathrm{n}=139$ ) necessita de aplicar medidas de contenção mecânica frequentemente ou muito frequentemente, corroborando o estudo de Hamers \& Huizing (2005). A regularidade da prática da contenção mecânica exige o reforço na formação dos enfermeiros.

Considerando a contenção mecânica não como uma técnica de eleição, mas como uma necessidade, os enfermeiros raramente referem intercorrências da sua aplicação (18\%; n=45). Destas, a mais frequente é o desenvolvimento de "solução de continuidade", seguida de "incompreensão dos familiares" e "equimose".

Relativamente à reavaliação da necessidade de contenção mecânica, sensivelmente metade (44\%; $\mathrm{n}=110$ ) efetua-a até uma hora após a sua aplicação e um quarto $(24,4 \% ; n=61)$ ao fim de duas horas e apenas $12 \%(n=30)$ demora 8 horas ou mais para a reavaliar.

O facto de dois terços $(66,8 \% ; n=167)$ dos enfermeiros referirem o recurso a técnicas preventivas à contenção revela uma preocupação em evitar esta medida. Das técnicas utilizadas, destacam-se o "diálogo com o doente", a "contenção química" e a "contenção ambiental". Esta realidade vai de encontro às recomendações da DGS (2011) para medidas alternativas ou de prevenção e reflete o aumento do seu emprego relativamente aos $20 \%$ verificados por Faria, Paiva \& Marques (2012).

Os questionários incluíram questões sobre os pensamentos associados à contenção mecânica sobre três perspetivas - a do enfermeiro, a do doente e a da família. Uma vez que a múltipla associação livre de palavras sem restrição de resposta gerou na ordem de 200 expressões diferentes em cada pergunta, concentramos a nossa discussão nas dez expressões mais referidas e que representam cerca de $40 \%$ em cada questão.

Quando pensam em contenção mecânica, os enfermeiros lembram-se de segurança, imobilização e agitação. Das dez expressões mais referidas, quatro remetem para a garantia da qualidade de cuidados (segurança, prevenção, necessidade, proteção), quatro descrevem a contenção (imobilização, amarrar, pulseira, imobilizador) e duas justificam-na (agitação, desorientação).

Questionados sobre os pensamentos associados aos doentes sujeitos à contenção mecânica, os enfermeiros referem principalmente segurança, necessidade e prevenção. Dos dez mais descritos, passam a ser sete os que se direcionam para a qualidade de cuidados (segurança, necessidade, 
prevenção, proteção, preocupação, decisão, vigilância) e três justificam a contenção (último recurso, exaustão, risco).

Por fim, na descrição dos pensamentos associados aos familiares dos doentes submetidos a contenção mecânica, os enfermeiros retratam seis pensamentos negativos (tristeza, incompreensão, revolta, medo, angústia, preocupação), dois positivos (compreensão, necessidade) e dois que induzem à necessidade de diálogo (explicação, informação).

Verificamos que os pensamentos dos enfermeiros desenvolvem-se no sentido de cumprimento do seu dever, salvaguardando a segurança do doente, descrevendo a razão da contenção, a forma com é aplicada e o porquê de ser instituída.

$\mathrm{Na}$ resposta aos pensamentos na perspetiva dos doentes, os enfermeiros enfatizam expressões que visam garantir a qualidade dos cuidados, distanciando-se de pensamentos negativos que os doentes inevitavelmente vivenciam.

É sobre a perspetiva dos familiares que os enfermeiros mais percecionam pensamentos negativos, induzindoos à necessidade de diálogo para justificar a medida como garantia de cuidados de saúde em situações de exceção. Este enfoque revela que os enfermeiros valorizam o pensamento dos familiares, quando uma contenção mecânica é instituída.

\section{CONCLUSÃO}

Foi sobre os pensamentos que ocorrem aos enfermeiros no processo de instituir uma contenção mecânica que esta investigação incidiu, procurando identificar a representação social da contenção mecânica para este grupo profissional. A ambivalência de pensamentos entre o direito à autodeterminação $\mathrm{e}$ a necessidade de decidirem o que é melhor para o doente, geram inquietações que pretendíamos aprofundar.

O nível de conhecimento dos enfermeiros sobre contenção mecânica foi esclarecido e a sua prática foi analisada. Como representação social concluímos que os enfermeiros praticam frequentemente a contenção mecânica por segurança, mas esforçam-se por evitála. Dão valor aos pensamentos dos familiares, mas distanciam-se dos pensamentos dos doentes submetidos a esta intervenção.

Relativamente às limitações deste estudo, identificamos a escassez de referências nacionais. Consideramos que o curto período de colheita de dados limitou a amostra, e, por conseguinte, uma maior representatividade da população. A compreensão do fenómeno da contenção mecânica é também limitada pelo facto do estudo se centrar na perceção dos enfermeiros e não na análise real da prática, com recolha de dados dos processos clínicos. $O$ estudo identificou uma divergência entre a formação dos enfermeiros e a efetividade da prática da contenção mecânica, remetendo para a necessidade de futuro investimento na implementação de protocolos hospitalares e divulgação da orientação da DGS.

\section{REFERÊNCIAS BIBLIOGRÁFICAS}

Choi, E., \& Song, M. (2003) - Physical restraint use in a Korean ICU. Seoul. Journal of Clinical Nursing. 12(5), 651-659.

Código Deontológico dos Enfermeiros (2015). Estatuto da Ordem dos Enfermeiros Lei n.o 156/2015. In Portal Ordem dos Enfermeiros. Recuperado em 10 de 
fevereiro de 2019, em https://www.ordemenfermeiros.pt/arquivo/legislaca o/Documents/Legislac aoOE/CodigoDeontologico.pdf

DGS. Direção Geral da Saúde. (2007. Medidas preventivas de comportamentos agressivos/violentos de doentes - contenção física. Lisboa. Direção Geral da Saúde.

DGS. Direção Geral da Saúde. (2011). Prevenção de comportamentos dos doentes que põem em causa a sua segurança e sua envolvente. Lisboa. Direção Geral da Saúde.

Faria, H., Paiva, A., \&Marques, P. (2012). A restrição física da mobilidade- estudo sobre aspetos ligados à sua utilização com fins terapêuticos. Revista de Enfermagem Referência, III Série (6),7-16

Fletcher, P.C., Hirdes, J.P. (2010) - Risk factors restriction in activity associated with fear of falling among seniors within the community. Journal of Patient Safety. 6(3), 187-191

Hamers, J.P., \& Huizing, A. R. (2005) Why physical restraints in the elderly. Zeitschrift für Gerontologie und Geriatrie. 38(1), 19-25.

Kontio, R., Valimaki, M., Putkonen, H., Kuosmanen, L., Scott, A., \& Joffe, G. (2010) Patient restrictions,: Are there ethical alternatives to seclusion and restraint?, Nursing Ethics 17(1), 65-76. Retirado de http://www.sagepub.co.uk

Marchand, P., \& Ratinaud, P. (2011). L'analyse de similitude appliquée aux corpus textuels: les primaires socialistes pour l'élection présidentielle française. Universidade de Toulouse, pp. 687-699. Retirado de http://lexicometrica.univparis3.fr/jadt/jadt2012/Com
munications/Marchand,\%20Pascal\%20et\%20al.\%20\%20L\%27analyse\%20de\%20similitude\%20appliquee \%20aux\%20corpus\%20textuels .pdf

Martin, B., \& Mathisen, L. (2005). Use of physical restraints in adult critical care: a bicultural study. American Journal of critical care, 14(2), 133-142. Retirado de http://ajcc.aacnjournals.org

Monteiro, C. [et al.] (2004) O paciente que manifesta agressividade na intervenção psiquiátrica: a repressentação do enfermeiro. Escola Anna Nery Revista de Enfermagem. 8(3), 439-447.

Paes, M.P., Borba, L.O., \& Maftum, M.A. (2011) Contenção física de pessoas com transtorno mental: percepções da equipa de enfermagem. Revista Ciência, Cuidados e Saúde. 240-247.

Segatore, M., \& Adams, D. (2001) - Managing delirium and agitation in elderly hospitalized orthopaedic patients: Part 2 - Interventions. Orthopaedic Nursing. 20(2), 61-73.

Silva, S., Camargo, B., \& Padilha, M. (2011) A Teoria das Representações Sociais nas pesquisas da Enfermagem Brasileira. Revista Brasileira de Enfermagem, 64(5), 947-951. Retirado de www.scielo.br/pdf/reben/v64n5/a22v64n5.pdf

Streubert, H., \& Carpenter, D. R. (2013). Investigação qualitativa em enfermagem - Avançado o imperativo humanista (5 ed.). Lisboa: Lusodidacta.

Vilelas, J. (2017). Investigação: O Processo de Construção do Conhecimento ( $2 \circ$ ed.). Lisboa: Edições Sílabo. 